\title{
Duration differences between rare and common words and their implications for the interpretation of word frequency effects
}

\author{
CHARLES E. WRIGHT \\ Bell Laboratories, Murray Hill, New Jersey 07974
}

\begin{abstract}
The spoken durations of rare and common words were compared in three experiments. Depending upon the task and the amount of practice, rare words (with frequencies less than 3 per million) were spoken as much as $24 \%$ slower than common words (with frequencies greater than 100 per million), when the words were equated for number of letters. This difference was observed even when the memory and lexical access demands of the task were minimized, and it can be explained by differences in the phonetic constituents of the two classes of words. The existence of these phonetic differences has been previously reported by Landauer and Streeter (1973) and by Zipf (1935). These findings argue against the view that all effects on the processing of accurately perceived words that are correlated with frequency may be unambiguously ascribed to operations that involve secondary memory (although this may be true of frequency effects per se). One recent experiment (Watkins, 1977) is examined in light of these findings.
\end{abstract}

The hypothesis that word frequency effects in memory experiments are localized in secondary memory comes originally from our intuition that this is how word frequency should manifest itself. The frequency of occurrence associated with a word is a feature that must be apprehended over time. Since primary memory is by definition transitory, differential effects of long. term experience must be represented in secondary memory.

It has been argued (e.g., Crowder, 1976, pp. 132-151; Glanzer \& Cunitz, 1966) that empirical support for this hypothesis can be found in the demonstrations that common words from initial list positions are recalled better in free and serial recall than rare words in the same list positions (Raymond, 1969; Sumby, 1963; Watkins \& Watkins, 1977). This argument requires the assumption that recall of the initial words in a list depends primarily on secondary memory. The effect of word frequency is presumed to be confined to secondary memory, since the studies cited above did not find a significant advantage for common words presented in other list positions.

A flaw with this hypothesis about the locus of word frequency effects is that it overlooks the possibility that differential effects of long-term experience may also be represented in an external memory, the language itself. Because of this possibility, we must differentiate between frequency effects associated with an individual's long-term experience and frequency effects associated with other aspects of words (such as their constituent

The author would like to thank T. K. Landauer, N. H. Macdonald, D. Rosenbaum, P. Somers, and S. Sternberg for their useful comments on earlier drafts of this paper. phonemes) that have become correlated with word frequency.

Several researchers have reported differences between common and rare words other than their frequency of usage. Landauer and Streeter (1973) have shown that: (1) after equating number of letters, rare words have more phonemes per word than do common words; (2) rare words and common words differ in phonemic and graphemic composition; (3) common words are more like other common words and rare words are more like other rare words when this similarity is measured by examining the set of words that can be created by replacing a single grapheme in an original word (as Anderson, Note 1, showed for phonemes). Frederiksen (1971), following Zipf (1935), has shown that it is the more "complex" phonemes that occur more often in rare words than in common words.

These researchers have been primarily interested in word recognition, and they have shown that these factors can explain some of the superiority of common words in perceptual recognition tasks. One might wish to argue, however, that in most memory experiments performance is not constrained by perceptual differences between the stimuli. According to this argument, the effects of the factors cited above will be negligible for memory experiments, and the assumption that common and rare words are equivalent in respects other than frequency of usage is still useful.

In this paper I show that the spoken durations of rare words are longer than those of common words. This relation might have been predicted from the findings cited above, since one might expect words with more phonemes or words with more complex phonemes to have longer durations. This expectation is strengthened 
by the finding that the duration of words in prose passages read aloud (controlled for the number of syllables and phrase boundary type, but not for number of letters) decreases with increasing frequency (Coker, Umeda, \& Browman, 1973; Umeda \& Quinn, Note 2), although other factors may be involved in this experimental situation.

The existence of a relation between word frequency and word duration bears on the interpretation of word frequency effects in those memory experiments in which subjects rehearse, or use a store that has a time-limited capacity, or a store in which information is lost with the passage of time. Consider, for example, the frequency effect in free recall mentioned above (Raymond, 1969). It has been shown that the probability of recall for each word in the initial portion of a list to be recalled is well predicted by the number of times that the word was rehearsed (Rundus, 1971). If a list of rare words takes longer to say than a list of common words, then each word will be rehearsed fewer times in a fixed period of time. Therefore, instead of being the result of a direct effect on secondary memory, the frequency effect in the initial part of a free recall list might result from an indirect effect of the number of rehearsals.

Three experiments were conducted to assess the degree to which word frequency and word duration are related, when the relation is measured under conditions more similar to those of a typical memory experiment than is the reading of prose passages. Two initial, informal experiments measured the duration of common and rare words following methods of Baddeley, Thomson, and Buchanan (1975). The first experiment provided measurements of the rate at which a long list of words can be read aloud: reading rate. The second experiment provided measurements of the rate at which a short, well-learned list of words can be repeated aloud: articulation rate. The third experiment provided measurements of the durations of words measured with the words spoken alone and in simple carrier phrases, conditions chosen to eliminate lexical access and minimize the memory load during production of the phrase.

\section{EXPERIMENT 1: RATE OF READING LONG LISTS ALOUD}

\footnotetext{
Method

Materials. Six sets of one-syllable words were selected from the Kucera and Francis (1967) word count. Three of the word sets were composed of common words (frequencies of 100 per million or greater) with lengths of three, four, and five letters, respectively, and three lists were composed of rare words (frequencies of 3 per million or less) with the same lengths in letters (hereafter referred to as letter length). One-syllable words with the proper letter length and frequency were excluded unless they met all of the following criteria: (1) They appeared in Webster's Seventh Collegiate Dictionary as words used in present-day American English; (2) they could not be decomposed into a stem and a simple ending (e.g., verbs ending in s); (3) their pronunciation was not ambiguous (e.g., lead); (4) they were not obscene. After the selection process, there remained 66 , 161 , and 89 common words and 80,251 , and 222 rare words with lengths three, four, and five letters.
}

For each subject six lists of $\mathbf{2 5}$ words were randomly selected, one from each of the word sets. These lists were typed doublespaced in capital letters in a single column on white paper.

Subjects. The subjects were six high school students from the Murray Hill, New Jersey, area. Three of the subjects were male and three were female. Subjects were paid $\$ 3$ for their services.

Procedure and Design. Reading rates were determined separately for each of the lists. The subject turned up the typed list and was given as much time to become familiar with it as he/she felt was required. Subjects did this by reading through the list, either silently or aloud, and asking questions about words of which they were unsure. Once the subject was ready the trial started. The subject first heard two noise hisses and then a 3,000-Hz beep, spaced at $750-\mathrm{msec}$ intervals. Upon hearing the beep the subject read the list aloud as fast as possible while attempting to pronounce all words correctly. Subjects were instructed that if a mispronunciation occurred (a rare event, never occurring more than once per trial, but about which no data were recorded), they were to continue reading. The duration of an utterance was measured excluding the latency from the tone until the subject's first sound.

Each subject read each list twice. The common and rare word lists for each length were always tested in immediate succession. The order of the common and rare word lists and of lists of each letter length was balanced separately across subjects.

Equipment. Subjects were tested individually. They were seated comfortably in a soundproofed booth wearing "openstyle" headphones with an attached microphone. The headphones rested lightly on the ears and did not distort the subjects' perceptions of their speech productions. Durations of the speech productions were measured in units of $10 \mathrm{msec}$ with a voiceactuated clock.

\section{Results and Discussion}

The results of this experiment are given in Figure 1. The mean duration of a rare word was $50.3 \pm 11.5 \mathrm{msec}$ (the mean plus or minus the standard error of the mean

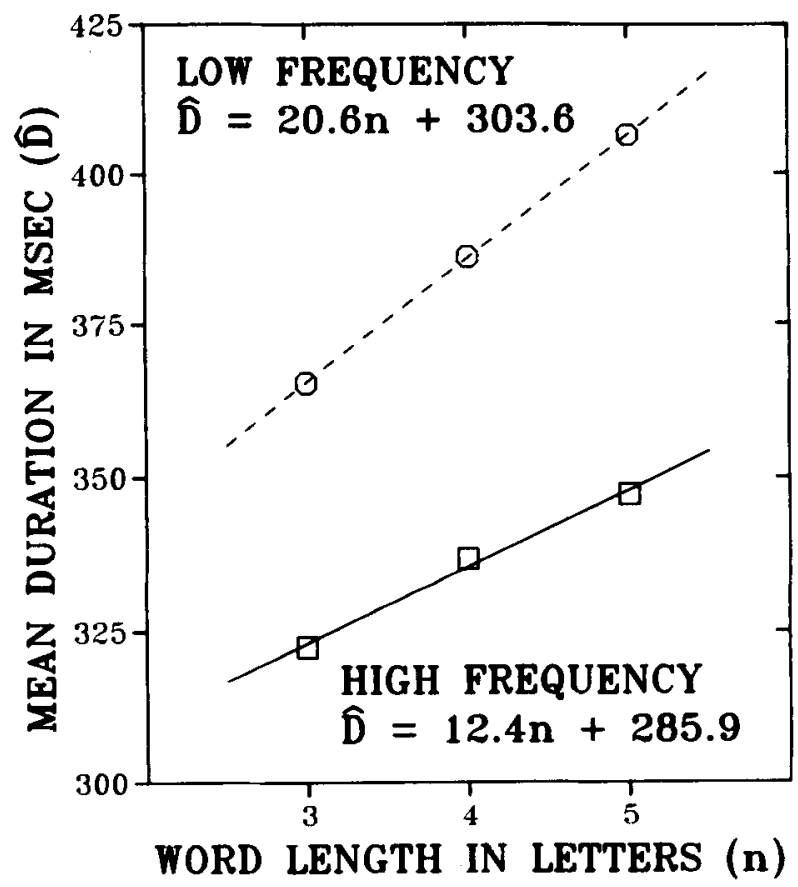

Figure 1. Mean duration of a word read from lists of words in Experiment 1. The results are averaged over six subjects and 25 words for each point. Also shown are the best-fitting linear functions. 
computed across subjects), or $15 \%$ longer than that of a common word $\left[\mathrm{F}(1,5)=19.28, \mathrm{p}<.01, \omega^{2}=26.7 \%\right]$. There was an effect of the length of a word (in letters) on mean duration $\left[\mathrm{F}(2,10)=6.60, \mathrm{p}<.025, \omega^{2}=\right.$ $4.3 \%]$. The best-fitting linear function relating duration to length had a slope of $16.5 \pm 2.7 \mathrm{msec} /$ word. Moreover, this slope was $8.2 \pm 2.8$ greater for rare words than for common words $[\mathrm{t}(5)=2.95, \mathrm{p}<.025]$.

\section{EXPERIMENT 2: RATE OF ARTICULATION FROM SHORT, WELL-LEARNED LISTS}

\section{Method}

Materials. Two lists of five common words and two lists of five rare words were randomly drawn for each subject from the sets of four-letter one-syllable words described in Experiment 1. The words were drawn with the restriction that no word appear on both lists for one subject, or on one of the lists that the subject had used in Experiment 1. Each list was typed doublespaced in capital letters in a single column on white paper.

Subjects. The same six subjects that participated in Experiment 1 were tested in Experiment 2.

Procedure and Design. A subject first was given time to memorize one common word list and one rare word list. The subject then produced the high-frequency word list aloud three times in sequence as fast as possible without stopping. Errors in production, garbled words as opposed to word transpositions or omissions, were frequent (averaging as often as every other attempt for some subjects). When errors occurred, subjects were instructed to stop and begin the trial again. Once a trial was correctly performed with the high-frequency word list, the subject switched to the low-frequency word list. This procedure was repeated 10 times. The subject was then given the two remaining lists to be memorized, and the testing process was repeated.

Equipment. The experiment was performed using the same equipment as in Experiment 1.

\section{Results and Discussion}

The results of Experiment 2 are shown in Figure 2. The mean duration of a rare word was $53.6 \pm 13.8 \mathrm{msec}$ (or $24 \%$ ) longer than that of a common word $[\mathrm{F}(1,5)=$ $15.05, \mathrm{p}<.025, \omega^{2}=26.4 \%$ ]. The mean durations of the rare words and the common words both decreased with practice $\left[F(9,45)=29.8, p<.001, \omega^{2}=21.6 \%\right]$; however, the mean duration of the rare words decreased faster $\left[F(9,45)=2.39, p=.025, \omega^{2}=1.1 \%\right]$. The effect of frequency was significant $(\mathrm{p}<.05)$ for each trial separately, and also for the effect computed based on the fastest time for each subject on each list. The correlations over subjects of the size of the frequency effect across experiments was $r=.24$. However, this ignores the fact that each subject used different materials in the two experiments, and so it may be an underestimate.

Taken together Experiments 1 and 2 provide evidence that word duration is associated with word frequency. Since the effect of frequency on word duration is of similar magnitude in two experiments with different memory and perceptual demands, it is tempting to conclude that this effect is not produced by processes associated with memory and perception. It is, however, still possible that some process common to both experiments, in which the lexical entry (or perhaps a motor program) is accessed, is responsible for the observed

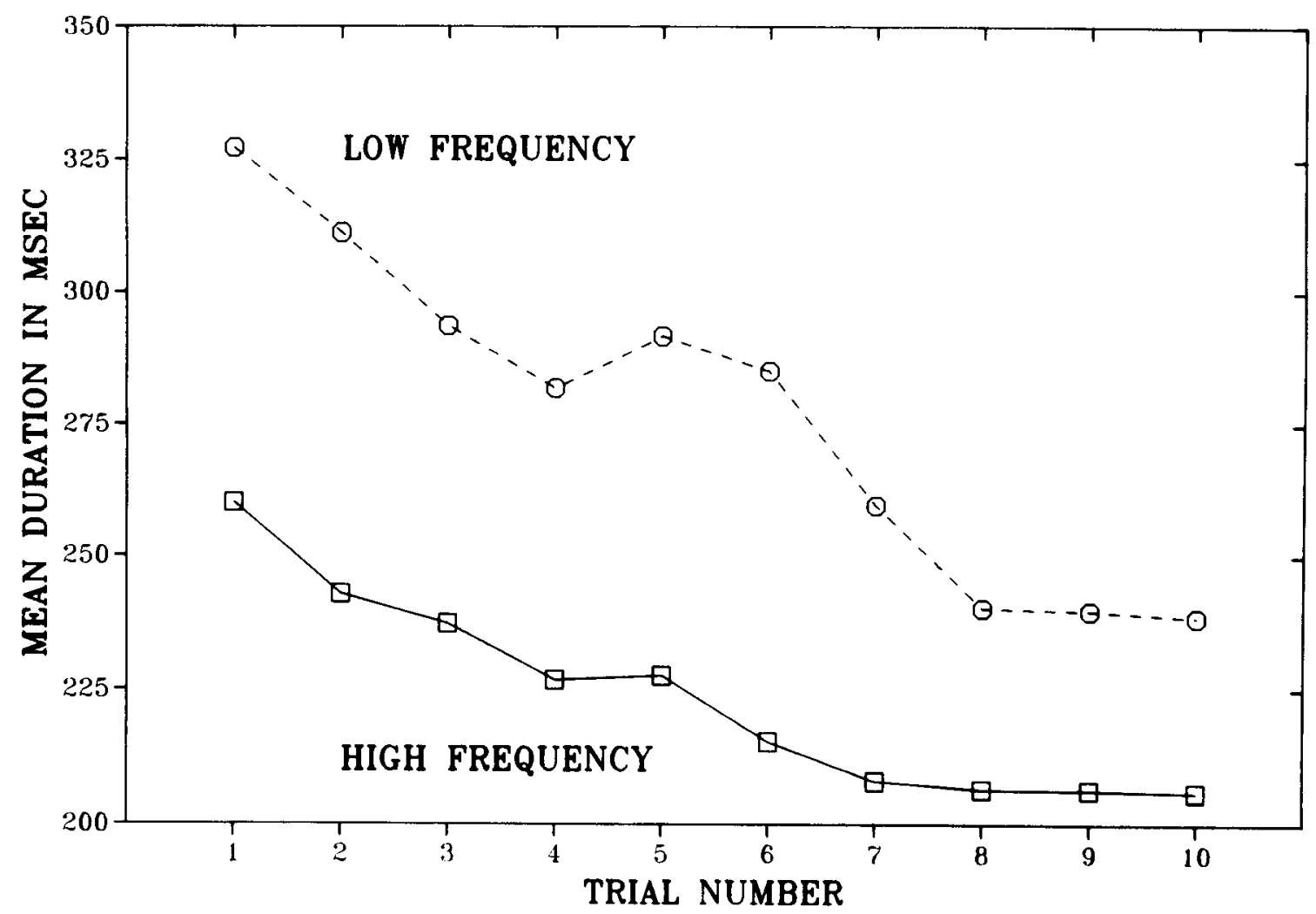

Figure 2. Mean duration of a word spoken from short, memorized lists in Experiment 2. The results for each point are averaged over six subjects, each performing three repetitions of two lists containing five words each. 
effect. To eliminate this possibility, an experiment in which short utterances are produced after ample preparation is required. Such an experiment might reduce the need for lexical access during the production. Since the utterances are short and phonetically well defined, it should also be possible to test the hypothesis that the effect of word frequency on duration is phonetically mediated.

\section{EXPERIMENT 3: DURATION OF WORDS SPOKEN IN ISOLATION OR SHORT PHRASES}

\section{Method}

Materials. For the third experiment, three sets of 36 words were constructed by repeatedly choosing, randomly without replacement, 6 words from each of the six sets described in Experiment 1. Thus each set of words contained 18 common words and 18 rare words evenly stratified by number of letters. An additional set of 18 words was similarly selected for use in the practice blocks.

Subjects. The subjects were six high school seniors or college freshmen from the Murray Hill, New Jersey, area. Three of the subjects were male and three were female. Subjects were paid $\$ 3$ for their services plus a small bonus determined by their performance. None had participated in Experiments 1 and 2 .

Design. The subjects were randomly grouped into three pairs. Each pair of subjects was tested on only one of the three sets of 36 words. The duration of each of the words in the set was measured twice in succession, at different times in the session for each of three conditions. The three conditions were a nocontext condition, in which the word was produced in isolation and two context conditions, in which the word was produced as part of a carrier phrase: "to at" in one condition and "go stop" in the other.

To maximize the precision of the duration estimates, the two context phrases were chosen so that the productions would begin and end with stop consonants. In addition, the context phrases were chosen to interact semantically as little as possible with the word being spoken.

The no-context condition was used with some trepidation, since there are reasons to believe that the durations of words produced in isolation differ from their durations in context. This is because, without a context, vowels and/or final stops of some words may be greatly lengthened. More generally, there are reasons to think that the production of words at the beginning or end of a list differs qualitatively from the production of words in interior list positions; therefore, comparison of the no-context with the context conditions may imply a false assumption of element invariance (see Sternberg, Monsell, Knoll, \& Wright, 1978 , p. 119). However, the no-context condition has the advantage of minimizing memory load. In addition this condition was of interest for reasons unrelated to this paper.

The trials were run in blocks of 18 . Since each word was measured in two successive trials, data for nine words were obtained in each block. Each context phrase was used for four successive blocks during which data were collected on each of the 36 words in the set for which the subject was being tested. The order of words within each context was randomized separately for each subject.

The four test blocks using a given context were preceded by a practice block using the same context and nine of the words from the practice set. The order in which the context phrases were administered was counterbalanced across subjects. The entire set of 15 blocks was preceded by an additional practice block using the context phrase "say fast" and the remaining nine words from the practice set. All 288 trials in this design were performed in less than $1 \mathrm{~h}$ by each subject.

Procedure. Subjects were comfortably seated in a soundproofed booth. They wore "open-style" headphones over which auditory signals were presented. These headphones rested lightly on the ears and did not distort the subjects' perceptions of their speech productions. Attached to the headphones was a microphone.

The sequence of events and the collection of data for each trial were computer controlled. On each trial subjects first heard a warning noise burst, followed $500 \mathrm{msec}$ later by a display on a CRT screen of the word or phrase to be produced on that trial. The display remained on the screen for $2.5 \mathrm{sec}$, during which subjects were encouraged to practice saying the phrase. After the display had been off for $250 \mathrm{msec}$, a low-level, $300-\mathrm{Hz}$ square-wave tone was presented for $50 \mathrm{msec}$. The tone informed the subject to produce the phrase, starting any time in the next $5 \mathrm{sec}$. Subjects were instructed that they need not begin speaking quickly after the tone, but that they should not make any extraneous noises once they had heard the tone. Subjects were asked to finish speaking the word or phrase as soon as possible after they began speaking, consistent with correct pronunciation.

The duration of a subject's entire production, including the carrier phrase, was the dependent variable. The duration was measured with a software speech detector in units of $10 \mathrm{msec}$. The subject's performance was monitored by the experimenter. Trials with incorrect pronunciations were counted as errors. The experimenter told the subject the correct pronunciation, and the trial was immediately repeated. (This action was required on $.7 \%$ of the test trials.) The experimenter monitored the productions on the screen of a storage oscilloscope for signs of any features of the speech production (such as breathing or lip noises) that might cause inaccurate measurement of the duration of the phrase by the software speech detector. These trials were not counted as errors, but they were immediately repeated. (This action was taken on .5\% of the test trials.) One second after the completion of a trial, the next trial in the sequence began.

After each block of 18 trials subjects were given feedback concerning their performance on that block. The feedback consisted of the average duration of their utterances in hundredths of a second and a score that was the sum of their average time and five points for each error. Subjects were instructed to try to minimize their scores and were given a 5 -cent bonus for each block in which their scores were equal to or better than the previous block in that context condition.

\section{Results and Discussion}

The results of this experiment are summarized in Figure 3. (Note that the abscissa of this figure is number of phonemes, rather than number of letters, which is listed beside each point, since number of phonemes will prove later to be a more powerful explanatory variable.) Since it was expected that the data from the no-context condition might be different from the data in the two context conditions, a separate analysis of variance was performed for each condition. These sixfactor analyses had three fully crossed fixed effects: frequency, length (in letters), and an experimental grouping factor (subject pairs). There were also three random factors: (1) subjects, which were nested in the groups and crossed with frequency and length,(2) words, which were nested within groups, frequency, and length, but crossed with subjects, and (3) replications, which were nested within all the other factors.

Since there is more than one random effect in this design, several of the tests could not be performed using the standard $\mathrm{F}$ ratio. In these cases, the quasi- $\mathrm{F}$ ratio (Winer, 1971, pp. 375-378) was used. The use of this test will be indicated by the symbol $F^{\prime \prime}$ instead of the symbol F. 


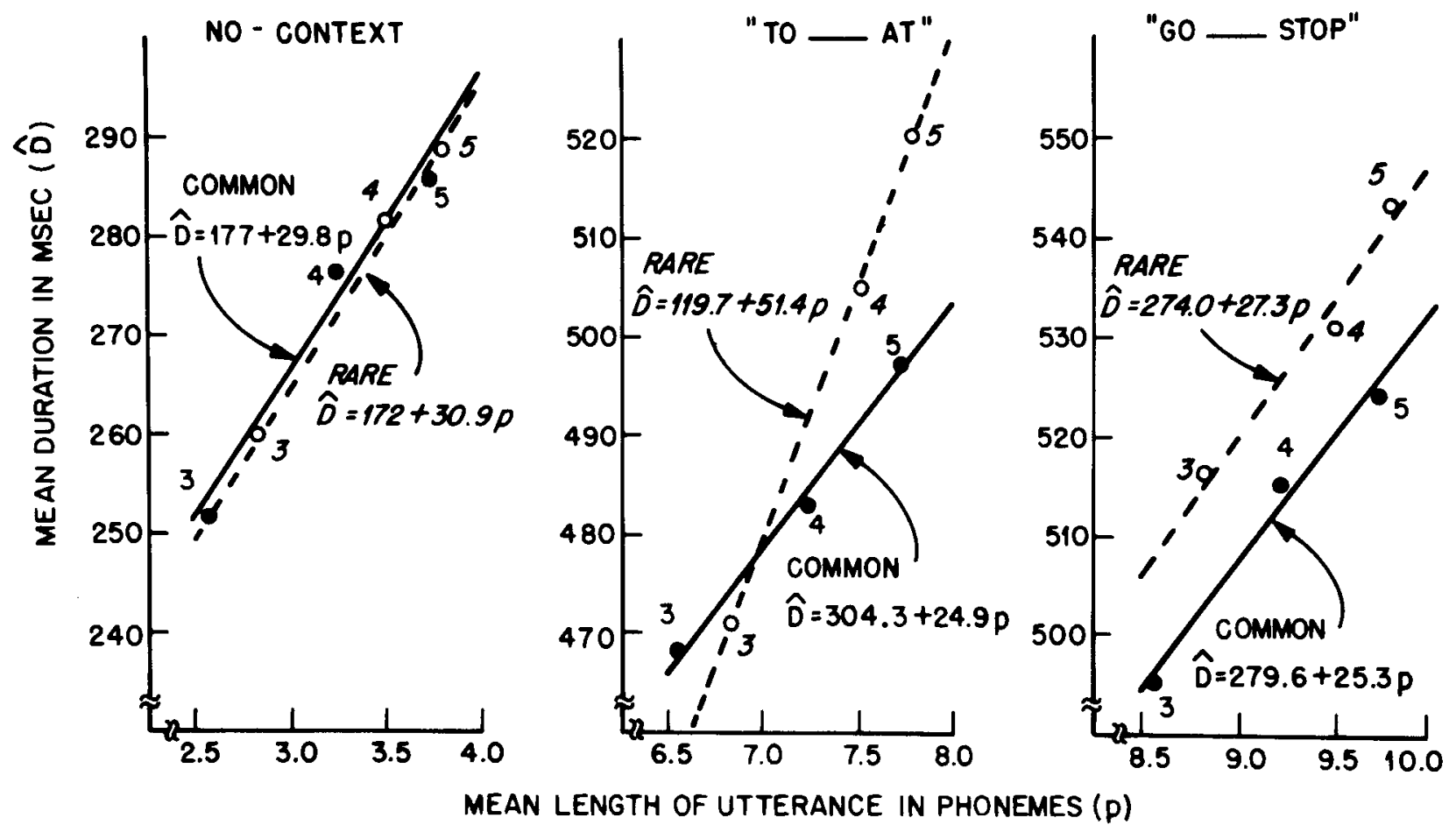

Figure 3. Mean duration of short phrases consisting of a two-word fixed context (null in the no-context condition) and a varied test word (test words are balanced across conditions). The results are averaged over two repetitions of six phrases by six subjects for each point. Also shown are the best-fitting linear functions.

Effects of frequency and letter length. Both of the context conditions showed significant main effects of frequency and length. For the "to___at" context, the mean frequency effect was $19.3 \mathrm{msec}\left[\mathrm{F}^{\prime \prime}(1,60)=6.28\right.$, $\mathrm{p}<.025]$, and the mean length effect was $22.2 \mathrm{msec} /$ character $\left[F^{\prime \prime}(2,19)=4.41, p<.05\right]$. For the "go___stop" context, the mean frequency effect was $18.4 \mathrm{msec}\left[\mathrm{F}^{\prime \prime}(1,26)=6.45, \mathrm{p}<.025\right]$, and the mean length effect was $14.3 \mathrm{msec} /$ character $\left[\mathrm{F}^{\prime \prime}(2,35)=4.65\right.$, $\mathrm{p}<.025]$.

In the no-context condition, neither of the main effects of interest was significant by a quasi- $F$ test. The mean frequency effect was $5.6 \mathrm{msec}\left[\mathrm{F}^{\prime \prime}(1,48)=.47\right]$, and the mean word length effect was $15.6 \mathrm{msec} / \mathrm{character}$ $\left[F^{\prime \prime}(2,45)=2.29, p>.10\right]$. However, when only error due to subjects was included in the error term, the length effect in the no-context condition was significant $[F(2,6)=6.02, p<.05]$.

An overall analysis of variance that included context condition as a factor showed that the differences among the three conditions in the sizes of the frequency and length effects were not significant [for the interaction of context with frequency, $F^{\prime \prime}(5,113)=1.44, p>.20$; for the interaction of context with word length, $\left.F^{\prime \prime}(21,45)=.62\right]$. What was significantly different among the three conditions was the effect of words. This was indicated by a significant Context by Words interaction $[F(180,180)=1.40, p<.01]$

This pattern of results is due in part to large differences among the three conditions in the variance components due to words $\left(\sigma_{\mathrm{w}}^{2}\right)$ and the Subjects by Words interaction $\left(\sigma_{S \times w}^{2}\right)$. (Computed within frequency, length, and subject pairs, the variance components for the three conditions were: "to _at," $\sigma_{w}^{2}=352, \sigma_{\mathrm{s} \times w}^{2}=$ 2,997; "go__stop," $\sigma_{\mathrm{w}}^{2}=118, \sigma_{\mathrm{s} X \mathrm{w}}^{2}=1,901$; nocontext, $\sigma_{\mathrm{w}}^{2}=2,255, \sigma_{\mathrm{SXw}}^{2}=865$.) This pattern of results indicates that the words in the no-context condition are produced in a way different from those in the two context conditions. Since the situations in memory experiments generally involve words in contexts, I feel that the large differences between the no-context and the context conditions make the no-context condition less relevant for the purposes of this paper.

Comparison with frequency effects in Experiments 1 and 2. Even in the context conditions the frequency effect was smaller in Experiment 3 (about $19 \mathrm{msec}$ ) than in Experiments 1 and 2 (50 and $54 \mathrm{msec}$, respectively). There are at least two interesting explanations of this difference. (1) While we may conclude that we successfully eliminated the perceptual processing of the visual image and the subsequent searching for the word in secondary memory during the productions in Experiment 3 , these processes, if they were necessary and could not be performed in parallel with the productions, may have contributed significantly to the size of the frequency effect in Experiments 1 and 2. (Frederiksen and Kroll, 1976, have reported frequency effects on word-naming latency of between 25 and $40 \mathrm{msec}$, depending on the experimental conditions. Cosky, 1976, has also reported a 41-msec effect. On the other hand, Richardson, 1976, and Scarborough, Cortese, and Scarborough, 1977, found no effects of frequency on 
word-naming latency.) (2) It is possible that when subjects can concentrate their attention on a very short utterance they are able to optimize difficult articulatory segments so as to reduce the incremental duration associated with rare words.

Phonemic correlates of the frequency and length effects. Can the frequency and length effects be explained in terms of the phonemes of the words involved? This question has two parts: (1) Are there more phonemes per word in rare than in common words (with the same letter length) or, alternatively, in words with more letters (and the same frequency)? (2) Are there more phonemes with longer durations in rare than in common words or, alternatively, in words with more letters?

The first part of this question has been answered affirmatively by Landauer and Streeter (1973). From Figure 3 it is clear that their results are confirmed in this experiment, since for each letter length (indicated by the numbers next to each point) the mean phoneme length (read from the abscissa) of the rare words is greater than that of the common words. [For the linear component of this trend, $\chi^{2}(1)=5.47, p<.025$; the nonlinear component of this trend was not significant, $\chi^{2}(1)=.03$.] From Figure 3 it is also clear that the number of phonemes in a word is closely related to its length in letters [linear component, $\chi^{2}(1)=87.80$, $\mathrm{p} \ll .001$; nonlinear component, $\left.\chi^{2}(3)=14.53, \mathrm{p}<.01\right]$.

That rare words contain more phonemes per word than do common words does not by itself explain the duration difference between them. It is also necessary to know the relative durations of the phonemes in rare and common words, since it is conceivable that differences in the durations might offset the effect of the number of phonemes. Looking at Figure 3, we note that the fitted lines in the two context conditions are not colinear, indicating that the mean duration of phonemes in the rare words must be longer than the mean duration of phonemes in the common words.

To ask whether this increase in mean duration is a general one, or whether it is due to a higher concentration of phonemes with long durations in rare words, we must first obtain estimates of the duration of each phoneme. These estimates were obtained separately for each condition and for both common and rare words, using multiple linear regression with the model:

$$
\mathrm{d}_{\mathrm{jk}}^{\mathrm{c}}=\mathrm{s}_{\mathrm{j}}^{\mathrm{c}}+\sum_{\mathrm{i}=1}^{35} \alpha_{\mathrm{ik}} \mathrm{P}_{\mathrm{i}}+\epsilon_{\mathrm{jk}}
$$

Here, $d_{j k}^{c}$ is the observed duration of word $k$ for subject $j$ in condition $c ; s_{j}^{c}$ is the mean over all words for subject $j$ in condition $c ; P_{i}$ is the estimate of the duration of phoneme $\mathrm{i} ; \alpha_{\mathrm{jk}}$ is a structural variable indicating the number of times phoneme $\mathrm{i}$ occurs in word $\mathrm{k}$ (all phonetic spellings were obtained from Kenyon \& Knott, 1953); and $\epsilon_{\mathrm{jk}}$ is error. The estimates of the phoneme durations, $P_{i}$, obtained for the common and rare words were then averaged together for each condition sepa- rately, to give estimates that were unbiased with respect to frequency. This process resulted in three sets of estimated phoneme durations.

Note that this simple concatenation model involves two assumptions concerning differences among phoneme durations, that can at best be regarded as approximations: (1) They are independent of the phoneme's position in the word and of its context within the word and among surrounding words (Umeda, 1975, 1977), and (2) they do not depend on the speaker.

To facilitate further analysis, the phonemes were then combined into five groups, separately for each context. Each group spanned one-fifth of the estimated range of durations between the very short and the very long phonemes for that context. (The ranges were: "to__at," $191 \mathrm{msec}$; "go___stop," $92 \mathrm{msec}$; and no-context, $180 \mathrm{msec}$.)

In the two context conditions there were significantly more of the shortest phonemes in the common words and more of the longest phonemes in the rare words [for the "to___at" condition, $\chi^{2}(1)=4.19$, $\mathrm{p}<.05$; for the "go___stop" condition, $\chi^{2}(1)=7.94$, $\mathrm{p}<.005]$. There was, however, no reliable linear trend in these conditions and no effect in the no-context condition.

We can also break down these estimated phoneme durations by letter length. Here, there were significant trends, such that longer phonemes tended to be found in words with more letters. [The linear components of the trend in the three conditions were: "to_at," $\chi^{2}(1)=5.18, \mathrm{p}<.025 ;$;go__stop," $\chi^{2}(1)=15.71$, $\mathrm{p}<.001$; no-context, $\chi^{2}(1)=9.53, \mathrm{p}<.005$.] The nonlinear component was significant only in the nocontext condition $\left[\chi^{2}(7)=21.42, p<.005\right]$.

Having estimated the durations of the individual phonemes, we may ask what size of letter length and frequency effects are accounted for by the linear concatenation model. To the extent that this simple model, which does not include any parameters for word frequency, can predict the frequency effects on word duration, we may conclude that these effects are phonetically mediated. These predictions are shown in Table 1, along with the estimates of the effects observed in Experiment 3.

The final column of Table 1 shows the ratio of the

Table 1

Fitted vs. Observed Effects of Word Frequency and Letter Length and the Between-Words Variance Accounted for, by Condition, in Experiment 3

\begin{tabular}{lrrrrrr} 
& \multicolumn{2}{c}{$\begin{array}{c}\text { Mean } \\
\text { Frequency }\end{array}$} & & \multicolumn{2}{c}{$\begin{array}{c}\text { Mean Letter } \\
\text { Length }\end{array}$} \\
\cline { 2 - 3 } Condition & $\mathrm{O}$ & $\mathrm{P}$ & & $\mathrm{O}$ & $\mathrm{P}$ & VR \\
\hline to__at & 19.3 & 15.2 & & 22.2 & 17.1 & .87 \\
go__stop & 18.4 & 15.1 & & 14.3 & 13.2 & 1.08 \\
No Context & 5.6 & 16.1 & & 15.6 & 23.5 & .59 \\
\hline
\end{tabular}

Note $-O=$ observed, $P=$ predicted,$V R=$ between-words-variance ratio (see text). 
observed between-word variance (computed within frequencies and lengths) to the between-word variance of the residuals that resulted from fitting the linear concatenation model. To the extent that the model is applicable at the level of individual words, the betweenword variance of the residuals should be small relative to the between-word variance of the observed data. The fact that the ratios are close to unity rather than small indicates that, although this model may account for the frequency and length effects moderately well, it is not a good predictor of individual word durations.

\section{GENERAL DISCUSSION}

The important result of the three experiments described in this paper is that the duration of spoken, rare, one-syllable words is longer than that of spoken, common, one-syllable words, when they are selected to have equal letter lengths. At the level of individual words there are, of course, counterexamples: For instance, one rare word that was used in Experiment 3 was "clef," which had a consistently shorter duration than "song," a common word that was also used in this experiment. However, on the average, one would expect a randomly sampled group of rare words to have longer durations than a randomly sampled group of common words.

This effect is also not a negligible one. In the experiments reported here using one-syllable words with very high frequencies and one-syllable words with very low frequencies, the effect due to frequency ranged between 18 and $54 \mathrm{msec}$ (ignoring the no-context condition in Experiment 3). In Experiments 1 and 2 (where this computation is possible) this effect was $17 \%$ to $24 \%$ of the mean duration of the common words. As an example of just how big a difference an effect of this magnitude might make, consider that the mean time to produce a six-word list of common words is about the same as the mean time to produce a five-word list of rare words.

When one restricts memory load and the need for lexical access, word frequency per se might be expected to have no effect on word duration. If we accept this view, then the effect of word frequency on word duration that was observed under these conditions in Experiment 3 implies that some other attribute of common and rare words that is correlated with their frequency is the source of the effect. It was also observed in Experiment 3 that word frequency is correlated with the number of phonemes per word (replicating the results of Landauer \& Streeter, 1973) and with the durations of phonemes in words. Since the combination of these effects was sufficient to predict most of the frequency effect in the context conditions, we may conclude that these factors are the cause of the correla. tion between word frequency and duration.

\section{Implications for Studies of Word Frequency Effects}

The correlation of word frequency with word duration complicates the interpretation of frequency effects in experiments in which duration may be an important factor. Experiments of this type include situations in which some process is limited by time (such as rehearsal in the free recall experiments considered in the introduction), in which the capacity of a store is time-limited (as is the "working memory" posited by Baddeley \& Hitch, 1974; Baddeley et al., 1975) and in which the accuracy of stored information is affected by the delay between its reinstatements (as in the "reverberatory loop" model of Lee \& Estes, 1977). To make these ideas more explicit it may be useful to show how they apply to a particular recent study. As an example I have chosen the study by Watkins (1977) that originally stimulated my interest in these questions.

The thesis of Watkins (1977) paper is that memory span is the product of something more than a unitary process. In the discussion of his experiment Watkins considers that the dual processes involved may be primary and secondary memory. Based on previous research demonstrating that frequency is one of several variables that interact with serial position in the singletrial free recall procedure (Raymond, 1969; Sumby, 1963), Watkins chose to vary the frequency of words in the two halves of presented memory span lists orthogonally and to examine the effects on span recall for such lists. He theorized that an interaction of word frequency with serial position in the memory span task would be sufficient evidence to conclude that span recall requires an interpretation at least as complex as a twoprocess account. His data are reproduced in Table 2.

The data agree with one set of Watkins' (1977) predictions. There is a frequency effect in both list halves, and this effect is bigger for the first half than for the second. Most importantly, the memory span for lists with high-frequency words followed by lowfrequency words (HL) is significantly greater than for lists with low-frequency words followed by highfrequency words (LH).

The main effect of frequency can be explained in terms of the correlation between frequency and duration by reference to the results of Baddeley et al. (1975). They performed memory span experiments in which the durations of the words in the memory lists were varied across conditions, while other factors (including fre-

Table 2

Mean Memory Span for High-Frequency (HH), Low-Frequency (LL), and Mixed-Frequency (HL and LH) Word Lists (from Watkins, 1977)

\begin{tabular}{cccc}
\hline HH & HL & LH & LL \\
\hline 5.82 & 5.19 & 4.65 & 4.24 \\
\hline
\end{tabular}


quency) were fixed. They found that memory span was a linear function of the durations of their word lists, and they suggested that this was due to memory span being limited by the capacity of a time-limited working memory. In the experiments reported here we found that rare words had durations up to $24 \%$ longer than common words. Applying the linear function of Baddeley et al., we would expect Watkins (1977) to have found an overall effect of similar magnitude. Referring to Table 2, we find that the memory span in the $\mathrm{HH}$ condition is in fact about $20 \%$ larger than the memory span in the LL condition.

What we cannot account for in this manner is the difference between the HL and the LH conditions. It is, however, possible to explain this difference in terms of the duration differences between common and rare words, if one is willing to make one of several assumptions.

One possibility is that, after seeing the list, the subject rehearses it covertly ${ }^{1}$ before writing his response. It is easy to show that the average time between the presentation of an item and its recall within this covert rehearsal depends on the type of list. In order of increasing mean delay the lists would be: $H H, H L, L H$, and $L L$. If one assumes that the probability of recalling an item decreases monotonically with increasing delay between its presentation and recall, then the ordering of the average recall times is sufficient ${ }^{2}$ to account for the ordering of entries in Table 2.

A second possibility is that, during presentation of the list, the subject constantly rehearses all of the list that has already been presented, in a cyclical fashion. Here again the difference in the duration of common and rare words leads to a difference in the average number of times that each word in a list is rehearsed. In order of decreasing number of rehearsals the lists would again be: $\mathrm{HH}, \mathrm{HL}, \mathrm{LH}$, and $\mathrm{LL}$. If we assume that the probability of recalling an item increases monotonically with the number of times it has been rehearsed, then the ordering of the average number of rehearsals per item for the different conditions can account for the ordering of the entries in Table 2.

Our findings do not tell us that an explanation of Watkins' (1977) findings based on variations in word duration is necessarily to be preferred to an explanation based on variations in word frequency per se. These findings do, however, call into question the frequency explanation and, by implication, Watkins' conclusion that memory span is not the product of a unitary process.

It should also be pointed out that the two possibilities suggested above are by no means new. It was the aim of distinguishing models of precisely these kinds that led to a series of experiments that examined the effects of varying the distribution of item presentation times in lists presented for short-term recall (Corballis, 1966; Corballis \& Loveless, 1967; Schonfield \& Donaldson, 1966).

\section{REFERENCE NOTES}

1. Anderson, D. C. The number and nature of alternatives as an index of intelligibility. Unpublished doctoral dissertation, Ohio State University, 1962.

2. Umeda, N., \& Quinn, A. M. S. Word duration as a function of boundary perception and the frequency of occurrence of the word. Internal memorandum, Bell Laboratories, 1976.

\section{REFERENCES}

Baddeley, A. D., \& Hitch, G. Working memory. In G, H. Bower (Ed.), The psychology of learning and motivation (Vol. 8). New York: Academic Press, 1974.

Baddeley, A. D., Thomson, N., \& Buchanan, M. Word length and the structure of short-term memory. Journal of Verbal Learning and Verbal Behavior, 1975, 14, 575-589.

Chase, W. G. Does memory scanning involve implicit speech? In S. Dornic (Ed.), Attention and performance VI. Hillsdale, N.J: Erlbaum, 1977.

Coker, C. H., Umeda, N., \& Browman, C. P. Automatic synthesis from ordinary English text. IEEE Transactions on A udio and Electroacoustics, 1973, AU-21-3, 293-298.

Corballis, M. C. Rehearsal and decay in immediate recall. Quarterly Journal of Experimental Psychology, 1966, 20, 43-51.

Corballis, M. C., \& Loveless, T. The effect of input modality on short-term serial recall. Psychonomic Science, 1967, 7, 275-276.

Cosky, M. J. The role of letter recognition in word recognition. Memory \& Cognition, 1976, 4, 207-214.

Crowder, R. G. Principles of learning and memory. Hillsdale, N.J: Erlbaum, 1976.

Frederiksen, J. R. Statistical decision model for auditory word recognition. Psychological Review, 1971, 5, 481-486

Frederiksen, J. R., \& Kroll, J. F. Spelling and sound: Approaches to the internal lexicon. Journal of Experimental Psychology: Human Perception and Performance, 1976, 2, 386-393.

Glanzer, M., \& Cunitz, A. Two storage mechanisms in free recall. Journal of Verbal Learning and Verbal Behavior, 1966, $5,351-360$.

KenYon, J. S., \& KNotT, T. A. A pronouncing dictionary of American English. Springfield, Mass: Merriam, 1953.

Kučra, H., \& Francis, W. N. Computational analysis of present-day American English. Providence, R.I: Brown University Press, 1967.

Landauer, T. K. Rate of implicit speech. Perceptual and Motor Skills, 1962, 15, 646 .

Landauer, T. K., \& Streeter, L. A. Structural differences between common and rare words: Failure of equivalence assumptions for theories of word recognition. Journal of Verbal Learning and Verbal Behavior, 1973, 12, 119-131.

LEE, C., \& EsTES, W. K. Order and position in primary memory for letter strings. Journal of Verbal Learning and Verbal Behavior, 1977, 16, 395-448.

RAYMOND, B. Short-term storage and long-term storage in free recall. Journal of Verbal Learning and Verbal Behavior, 1969, 80, 95-100.

Richardson, J. T. E. The effects of stimulus attributes upon latency of word recognition. British Journal of Experimental Psychology, 1976, 67, 315-325.

Rundus, D. Analysis of rehearsal processes in free recall. Journal of Experimental Psychology, 1971, 89, 63-77.

Scarborough, D. L., Cortese, C., \& Scarborough, H. S. Frequency and repetition effects in lexical memory. Journal of Experimental Psychology: Human Perception and Performance, 1977, 3, 1-17.

Schonfield, D., \& Donaldson, W. Immediate memory as a function of intra-series variation. Canadian Journal of Psychology, 1966, 20, 218-227. 
Sternberg, S., Monsell, S., Knoll, R. L., \& Wright, C. E. The latency and duration of rapid movement sequences: Comparison of speech and typewriting. In G. Stelmach (Ed.), Information processing in motor control and learning. New York; Academic Press, 1978.

Sumby, W. H. Word frequency and serial position effects. Journal of Verbal Learning and Verbal Behavior, 1963, 1 , 443-450.

UMEDA, N. Vowel duration in American English. Journal of the Acoustical Society of America, 1975, 58, 434-445.

UMEDA, N. Consonant duration in American English. Journal of the Acoustical Society of America, 1977, 61, 848-858.

Watkins, M. J. The intricacy of memory span. Memory \& Cognition, 1977, 5, 529-534.

Watkins, O.C., \& W Atk Ins, M. J. Serial recall and the modality effect: Effects of word frequency. Journal of Experimental Psychology: Human Learning and Memory, 1977, 3, 712-718.

WINER, B. J. Statistical principles in experimental design. New York: McGraw-Hill, 1971.

$\mathrm{ZIPF}, \mathrm{G}$. K. The psycho-biology of language: An introduction to dynamic philology. New York: Houghton Mifflin, 1935.

\section{NOTES}

1. Strictly speaking the results of this paper cannot be directly applied to situations involving covert speech. However, claims have been made that covert speech is similar to overt speech in its durational properties (Chase, 1977, p. 613; Landauer, 1962). These claims agree with the preliminary results I have obtained using the techniques of Experiment 2. The data from two subjects indicate that the frequency effect is identical in overt and covert recitation.

2. This conclusion, here and in the next paragraph, is not strictly correct. If there is an overlap of the distributions of mean delay (or mean number of rehearsals) between the conditions, then there exist monotonic functions for which the ordering of the conditions on mean delay (or mean number of rehearsals) does not predict their ordering on memory span.

(Received for publication March 28, 1979; revision accepted September 28, 1979.) 